PROCEEDINGS OF THE AMERICAN MATHEMATICAL SOCIETY

Volume 124, Number 11, November 1996

\title{
AN ALGEBRAIC VERSION OF DEMAILLY'S ASYMPTOTIC MORSE INEQUALITIES
}

\author{
FLAVIO ANGELINI
}

(Communicated by Eric M. Friedlander)

\begin{abstract}
We give an elementary algebraic proof of some asymptotic estimates (called by Demailly asymptotic Morse inequalities) for the dimensions of cohomology groups of the difference of two ample line bundles on a smooth complex projective variety of any dimension.
\end{abstract}

\section{INTRODUCTION}

In [De1] Demailly proves the asymptotic Morse inequalities for a holomorphic line bundle on a compact Kähler manifold. His methods are essentially analytic and his first formulation involves some curvature integrals in general quite difficult to compute. A formulation of these inequalities in a more algebraic setting appears in [De2, §7]. Motivated by the work of Siu on an effective version of Matsusaka's big theorem [Siu] and by a work of Trapani on the difference of two ample line bundles [Tra], Demailly deduces, from his Morse inequalities, some asymptotic estimates for the dimensions of cohomology groups of the difference of two numerically effective line bundles in which only algebraic invariants are involved [De3, §12]. The purpose of this note is to give an elementary algebraic proof of these estimates in the case of a smooth projective variety.

The theorem is the following:

Theorem A. Let $L=F-G$ be a line bundle on a smooth complex projective variety $X$ of dimension $n$, where $F$ and $G$ are ample line bundles. Then, for every $q=0,1, \ldots, n$ and $k$ sufficiently large, we have:

$$
\begin{aligned}
h^{q}(X, k L) & \leq k^{n} \frac{F^{n-q} \cdot G^{q}}{(n-q) ! q !}+o\left(k^{n}\right), \\
\sum_{i=0}^{q}(-1)^{q-i} h^{i}(X, k L) & \leq \frac{k^{n}}{n !} \sum_{i=0}^{q}(-1)^{q-i}\left(\begin{array}{c}
n \\
i
\end{array}\right) F^{n-i} \cdot G^{i}+o\left(k^{n}\right) .
\end{aligned}
$$

Demailly [De3, §12] calls (1) and (2) respectively weak and strong asymptotic Morse inequalities.

The case $q=1$ gives a lower bound for the dimension of the space of sections $h^{0}(X, k L)$ which was used by Siu [Siu] in the course of his proof of an effective

Received by the editors March 6, 1995 .

1991 Mathematics Subject Classification. Primary 14F99; Secondary 32J99.

(C)1996 American Mathematical Society 
version of Matsusaka's big theorem. An algebraic proof in this case was given by R. Lazarsfeld and F. Catanese [De3, §12].

More generally the theorem holds under milder positivity hypothesis on $F$, depending on $q$, i.e. when $F$ is ample off a set of dimension less than or equal to $q-1$.

Theorem B. Suppose $G$ is an ample line bundle and $F$ is a line bundle having the property that there exist an ample vector bundle $E$ and a surjective map

$$
E \longrightarrow \mathcal{I}_{V} \otimes F \longrightarrow 0
$$

where $\mathcal{I}_{V}$ is the ideal sheaf of a subset $V$. Then, for $k$ sufficiently large and $q \geq$ $\operatorname{dim}(V)+1$, the inequalities (1) and (2) of Theorem A hold for $L=F-G$.

Clearly Theorem B implies Theorem A.

As a consequence we can obtain asymptotic estimates for the dimensions of the cohomology groups of a big line bundle $L$. If some multiple of $L$ is written as $a L=A+D$, where $A$ is an ample divisor and $D$ an effective divisor, the upper bound is expressed in terms of the first Chern class of $L$, the multiplicities of the singular points of $D$ and the curvature of the tangent bundle $T X$ [De2, §7],[De3, $\S 12]$.

This note is part of my Ph.D. thesis at UCLA, and I would like to thank Rob Lazarsfeld for his guidance and encouragement. I would like also to thank J.-P. Demailly for his prompt and detailed correspondence about the background on the topic.

\section{Proof of Theorem B}

First we need the following:

Lemma. Let $F$ be a line bundle on a smooth projective variety $X$ satisfying the hypothesis of Theorem B. Then, for any very ample divisor $H$ on $X$, there exists $k_{o}=k_{o}(X, F, H)$ such that

$$
H^{q}(X, k F+a H)=0
$$

for $q \geq \max (1, \operatorname{dim}(V)+1), k \geq k_{o}$ and any $a \geq 0$. In particular there exists $k_{o}=k_{o}(X, F)$ such that $H^{q}(X, k F)=0$ for $k \geq k_{o}$ and $q \geq \max (1, \operatorname{dim}(V)+1)$.

Proof. If $\operatorname{dim}(V)=-1$, i.e. $V=\emptyset$, then $F$ is ample. This is true also for $\operatorname{dim}(V)=$ 0 [for any ample $A, S^{k} E \otimes \mathcal{O}_{X}(-A)$ is globally generated for $k$ sufficiently large]. But then, in these two cases, there exists $k_{o}=k_{o}(X, F)$ such that $k_{o} F=K_{X}+$ (ample), where $K_{X}$ is the canonical bundle of $X$. Hence $k F+a H=K_{X}+$ (ample) for $k \geq k_{o}$ and any $a \geq 0$ and the Lemma follows from Kodaira vanishing.

Now suppose $\operatorname{dim}(V)=m$ with $m \geq 1$. Pick a general smooth irreducible divisor $D$ in the linear series of $H$ that meets $V$ properly and consider, for $a \geq 1$, the short exact sequence

$$
0 \longrightarrow \mathcal{O}_{X}(k F+(a-1) H) \longrightarrow \mathcal{O}_{X}(k F+a H) \longrightarrow \mathcal{O}_{D}(k F+a H) \longrightarrow 0 .
$$

By induction $H^{q-1}\left(D, \mathcal{O}_{D}(k F+a H)\right)=0$ for $k \geq k_{o}(D, F, H), q \geq m+1$ and any $a \geq 0$. Hence

$$
H^{q}\left(X, \mathcal{O}_{X}(k F+(a-1) H)\right)=H^{q}\left(X, \mathcal{O}_{X}(k F+a H)\right)
$$

for any $a \geq 0$ and $k \geq k_{o}$. But, for large $a, H^{q}\left(X, \mathcal{O}_{X}(k F+a H)\right)=0$. 
To simplify notation let us set, for a divisor $B$ on $X$,

$$
\chi_{q}\left(X, \mathcal{O}_{X}(B)\right)=\sum_{i=0}^{q}(-1)^{q-i} h^{i}\left(X, \mathcal{O}_{X}(B)\right) .
$$

Observe that, if

$$
0 \rightarrow A \rightarrow B \rightarrow C \rightarrow 0
$$

is an exact sequence of line bundles, then

$$
\chi_{q}(X, B) \leq \chi_{q}(X, A)+\chi_{q}(X, C) .
$$

One can check that by writing the long exact sequence truncated at the $q-t h$ group, it follows that $\chi_{q}$ satisfies similar subadditivity with respect to filtrations.

Proof of Theorem B. Let us pick a positive integer $a$ such that $a G$ is very ample and fix a smooth irreducible divisor $D$ in this linear series meeting $V$ properly. In order to use induction on $\operatorname{dim}(X)$ we will first prove an analogue of the Theorem for the bundle $k F-j a G$ for some positive integer $j$. Subsequently we'll deduce the stated inequalities for $L=F-G$. Specifically, we'll prove:

$$
\chi_{q}\left(X, \mathcal{O}_{X}(k F-j a G)\right) \leq \frac{1}{n !} \sum_{i=0}^{q}\left[(-1)^{q-i} k^{n-i}(j a)^{i}\left(\begin{array}{c}
n \\
i
\end{array}\right) F^{n-i} \cdot G^{i}+o\left(k^{n-i}\right) o\left(j^{i}\right)\right] .
$$

We will only prove (2) since (1) is similar and simpler. To do so consider the exact sequence

$$
0 \longrightarrow \mathcal{O}_{X}(k F-j a G) \longrightarrow \mathcal{O}_{X}(k F) \longrightarrow \mathcal{O}_{j D}(k F) \longrightarrow 0 .
$$

We have a filtration of $\mathcal{O}_{j D}$ whose quotients are line bundles of the form $\mathcal{O}_{D}(-l D)=$ $\mathcal{O}_{D}(-l a G)$ with $l=0, \ldots, j-1$. From the Lemma we have $H^{q}\left(X, \mathcal{O}_{X}(k F)\right)=0$ for $k$ sufficiently large. Then from $(*)$ we get the long exact sequence in cohomology

$$
\begin{aligned}
0 & \rightarrow H^{o}\left(X, \mathcal{O}_{X}(k F-j a G)\right) \rightarrow H^{o}\left(X, \mathcal{O}_{X}(k F)\right) \rightarrow H^{o}\left(j D, \mathcal{O}_{j D}(k F)\right) \rightarrow \ldots \\
\rightarrow H^{q-1}\left(j D, \mathcal{O}_{j D}(k F)\right) \rightarrow H^{q}\left(X, \mathcal{O}_{X}(k F-j a G)\right) \rightarrow H^{q}\left(X, \mathcal{O}_{X}(k F)\right) & =0 .
\end{aligned}
$$

Therefore

$$
\chi_{q}\left(X, \mathcal{O}_{X}(k F-j a G)\right)=\chi_{q}\left(X, \mathcal{O}_{X}(k F)\right)+\chi_{q-1}\left(j D, \mathcal{O}_{j D}(k F)\right) .
$$

Now from the filtration of $\mathcal{O}_{j D}$, we have

$$
\chi_{q-1}\left(j D, \mathcal{O}_{j D}(k F)\right) \leq \sum_{l=0}^{j-1} \chi_{q-1}\left(D, \mathcal{O}_{D}(k F-l a G)\right) .
$$


Since the bundle $\mathcal{O}_{D}(F)$ satisfies the hypothesis of the theorem with $X$ replaced by $D$ and $V$ replaced by $V \cap D$, we can use induction and assume:

$$
\begin{aligned}
& \chi_{q-1}\left(D, \mathcal{O}_{D}(k F-l a G)\right) \\
& \leq \frac{1}{(n-1) !} \sum_{i=0}^{q-1}\left[\left.\left.(-1)^{q-1-i} k^{n-1-i} l^{i} a^{i}\left(\begin{array}{c}
n-1 \\
i
\end{array}\right) F\right|_{D} ^{n-1-i} \cdot G\right|_{D} ^{i}+o\left(k^{n-1-i}\right) o\left(l^{i}\right)\right] .
\end{aligned}
$$

Therefore, replacing $\left.\left.F\right|_{D} ^{n-1-i} \cdot G\right|_{D} ^{i}$ with $F^{n-1-i} \cdot G^{i} \cdot D=a F^{n-1-i} \cdot G^{i+1}$, we find:

$$
\chi_{q}\left(X, \mathcal{O}_{X}(k F-j a G)\right) \leq \chi_{q}\left(X, \mathcal{O}_{X}(k F)\right)+
$$

$\frac{1}{(n-1) !} \sum_{l=0}^{j-1} \sum_{i=0}^{q-1}\left[(-1)^{q-1-i} k^{n-1-i} l^{i} a^{i+1}\left(\begin{array}{c}n-1 \\ i\end{array}\right) F^{n-1-i} \cdot G^{i+1}+o\left(k^{n-1-i}\right) o\left(l^{i}\right)\right]$.

Using that $\sum_{l=0}^{j-1} l^{i}=\frac{j^{i+1}}{i+1}+o\left(j^{i+1}\right)$ and that, since $H^{i}\left(X, \mathcal{O}_{X}(k F)\right)=0$ for $i \geq$ $q, \chi_{q}\left(X, \mathcal{O}_{X}(k F)\right)=(-1)^{q} \chi\left(X, \mathcal{O}_{X}(k F)\right.$ ) (where $\chi$ is the usual characteristic of Euler-Poincaré) the right hand side of the above inequality is equal to

$$
\begin{aligned}
& (-1)^{q}\left[k^{n} \frac{F^{n}}{n !}+o\left(k^{n}\right)\right]+ \\
& \frac{1}{(n-1) !} \sum_{i=0}^{q-1}\left[(-1)^{q-1-i} k^{n-1-i} \frac{j^{i+1}}{i+1} a^{i+1}\left(\begin{array}{c}
n-1 \\
i
\end{array}\right) F^{n-1-i} \cdot G^{i+1}+o\left(k^{n-1-i}\right) o\left(j^{i+1}\right)\right] .
\end{aligned}
$$

Using the identity $\frac{n}{i+1}\left(\begin{array}{c}n-1 \\ i\end{array}\right)=\left(\begin{array}{c}n \\ i+1\end{array}\right)$ and reindexing, the above can be written as

$$
\frac{1}{n !} \sum_{i=0}^{q}\left[(-1)^{q-i} k^{n-i} j^{i} a^{i}\left(\begin{array}{c}
n \\
i
\end{array}\right) F^{n-i} \cdot G^{i}+o\left(k^{n-i}\right) o\left(j^{i}\right)\right]
$$

which is what we wanted.

The first step of induction reduces, by cutting with hyperplane sections, to the case $q=0(V=\emptyset)$ which works because

$$
\begin{aligned}
\chi_{0}\left(X, \mathcal{O}_{X}(k F-l a G)\right) & =h^{0}\left(X, \mathcal{O}_{X}(k F-l a G)\right) \\
& \leq h^{0}\left(X, \mathcal{O}_{X}(k F)\right)=k^{n} \frac{F^{n}}{n !}+o\left(k^{n}\right) .
\end{aligned}
$$

To finish the proof of Theorem B let us take a large $k$ and write it as $k=j a+a+r$, where $r<a$ and $a$ is chosen so that $m G$ is very ample for any $m \geq a$. Pick a smooth irreducible divisor $D \in|(a+r) G|$. In this case we'll show (1), since (2) is similar, but involving much heavier formulas. We have

$$
\begin{aligned}
h^{q}(X, & \mathcal{O}_{X}(k(F-G)) \leq h^{q}\left(X, \mathcal{O}_{X}(k F-j a G)\right)+h^{q-1}\left(D, \mathcal{O}_{D}(k F-j a G)\right) \\
\leq & k^{n-q}(j a)^{q} \frac{F^{n-q} \cdot G^{q}}{(n-q) ! q !}+o\left(k^{n-q}\right) o\left(j^{q}\right) \\
& +k^{n-q}(j a)^{q-1}(a+r) \frac{F^{n-q} \cdot G^{q}}{(n-q) !(q-1) !}+o\left(k^{n-q}\right) o\left(j^{q-1}\right) \\
& \leq k^{n} \frac{F^{n-q} \cdot G^{q}}{(n-q) ! q !}+o\left(k^{n}\right) .
\end{aligned}
$$

(2) works in the same way because

$$
\chi_{q}\left(X, \mathcal{O}_{X}(k F-G)\right) \leq \chi_{q}\left(X, \mathcal{O}_{X}(k F-j a G)\right)+\chi_{q-1}\left(D, \mathcal{O}_{D}(k F-j a G)\right) .
$$


Now we would like to give an idea of how one can use Theorem B to obtain similar results in the case of a big line bundle, under some positivity hypothesis for the tangent bundle of the variety. For more precise definitions and statements we refer to $[\mathrm{De} 2, \S 7]$ or $[\mathrm{De} 3, \S 12]$.

Let $X$ be a smooth projective variety, $L$ a big line bundle on $X$, and suppose there exists an ample line bundle $E$ such that $T X \otimes \mathcal{O}_{X}(E)$ is ample. Some multiple of $L$ can be written as $a L=A+D$ with $A$ ample and $D$ effective divisors and we can attach to $L$ some numbers $0=b_{1} \leq \ldots \leq b_{n+1}$ obtained by counting the multiplicities of the singular points of $D$.

If we let $J^{r}$ be the $r$-th jet bundle associated to $a L$, then we get, for $q=0, \ldots, n$, a surjective map

$$
J^{a b_{n-q+1}} \otimes \mathcal{O}_{X}\left(a b_{n-q+1} E\right) \longrightarrow \mathcal{I}_{V} \otimes \mathcal{O}_{X}\left(a L+a b_{n-q+1} E\right) \longrightarrow 0
$$

where $V$ is a locus of singular points of $D$ that turns out, by definition of the $b_{i}$ 's, to be of dimension less than or equal to $q-1$.

Now $J^{a b_{n-q+1}} \otimes \mathcal{O}_{X}\left(a b_{n-q+1} E\right)$ is ample so we can apply Theorem B to the bundles $F=a L+a b_{n-q+1} E$ and $G=a b_{n-q+1} E$ and obtain asymptotic estimates for the dimensions of the cohomology groups of $a L$. With further arguments we can actually obtain the same results for $L$ itself.

\section{REFERENCES}

[De1] J.-P. Demailly, Champs magnétiques et inégalités de Morse pour la d"-cohomologie, Ann. Inst. Fourier (Grenoble) 35 (1985), 189-229. MR 87d:58147

[De2] J.-P. Demailly, Singular hermitian metrics on positive line bundles, Conf. Complex algebraic varieties (Bayreuth, April 2-6, 1990), edited by K. Hulek, T. Peternell, M. Schneider, F. Schreyer, Lecture Notes in Math., Vol. 1507, Springer-Verlag, Berlin, 1992. MR 93g:32044

[De3] J.-P. Demailly, $L^{2}$ vanishing theorems for positive line bundles and adjunction theory, CIME session on Transcendental Methods in Alg. Geom.(Cetraro, Italy, July 1994), Prépublication de l'Inst. Fourier 288 (1994).

[Siu] Y. T. Siu, An effective Matsusaka big theorem, Ann. Inst. Fourier 43 (1993), 1387-1405. MR 95f:32035

[Tra] S. Trapani, Numerical criteria for the positivity of the difference of ample divisors, preprint (1991).

Département de Mathématiques, Université de Nice-Sophia-Antipolis, Parc Valrose, 06108 Nice, France

E-mail address: angelini@math.unice.fr 